\title{
Focused ultrasound-aided immunomodulation in glioblastoma multiforme: a therapeutic concept
}

\author{
Or Cohen-Inbar ${ }^{1,2^{*}}$, Zhiyuan $\mathrm{Xu}^{1}$ and Jason P. Sheehan ${ }^{1}$
}

\begin{abstract}
Patients with glioblastoma multiforme (GBM) exhibit a deficient anti-tumor immune response. Both arms of the immune system were shown to be hampered in GBM, namely the local cellular immunity mediated by the Th, subset of helper T cells and the systemic humoral immunity mediated by the $\mathrm{Th}_{2}$ subset of helper $\mathrm{T}$ cells. Immunotherapy is rapidly becoming one of the pillars of anti-cancer therapy. GBM has not received similar clinical successes as of yet, which may be attributed to its relative inaccessibility (the blood-brain barrier (BBB)), its poor immunogenicity, few characterized cancer antigens, or any of the many other immune mechanisms known to be hampered. Focused ultrasound (FUS) is emerging as a promising treatment approach. The effects of FUS on the tissue are not merely thermal. Mounting evidence suggests that in addition to thermal ablation, FUS induces mechanical acoustic cavitation and immunomodulation plays a key role in boosting the host anti-tumor immune responses. We separately discuss the different pertinent immunosuppressive mechanisms harnessed by GBM and the immunomodulatory effects of FUS. The effect of FUS and microbubbles in disrupting the BBB and introducing antigens and drugs to the tumor milieu is discussed. The FUS-induced pro-inflammatory cytokines secretion and stress response, the FUS-induced change in the intra-tumoral immune-cells populations, the FUS-induced augmentation of dendritic cells activity, and the FUS-induced increased cytotoxic cells potency are all discussed. We next attempt at offering a conceptual synopsis of the synergistic treatment of GBM utilizing FUS and immunotherapy. In conclusion, it is increasingly apparent that no single treatment modality will triumph on GBM. The reviewed FUS-induced immunomodulation effects can be harnessed to current and developing immunotherapy approaches. Together, these may overcome GBM-induced immune-evasion and generate a clinically relevant anti-tumor immune response.
\end{abstract}

Keywords: Focused ultrasound, FUS, HIFU, GBM, Immunomodulation, Synergistic immunotherapy

\section{Background}

Glioblastoma multiforme (GBM) is the most common primary malignant brain tumor in adults. Despite standard of care treatment, the median survival of a patient harboring a GBM is less than 2 years. Although subdivided to different subtypes, these overall grim prognosis figures have stood fast and changed very little in the past few decades, proving resistant to most developments and revolutions incurred on modern medicine [1-3].

\footnotetext{
* Correspondence: oc2f@virginia.edu

'Department of Neurological Surgery, University of Virginia, Charlottesville, VA, USA

${ }^{2}$ Molecular Immunology \& Tumor Immunotherapy Laboratory, Technion-Israel Institute of Technology, Haifa, Israel
}

Current day first-line treatment for GBM patients includes a combination of surgery, chemotherapy, and radiotherapy [1-3]. Recent understanding of the role of epigenetic inheritance (e.g., promoter methylation) adds complexity to this issue. $O^{6}$-methylguanine-DNA methyltransferase (MGMT) is a key repair enzyme that contributes to the resistance of tumors to alkylating agents (carmustine or temozolomide). MGMT promoter methylation silences the gene, thereby increasing the susceptibility of the tumor cells to these agents [1-3].

The unique nature of GBM and its inherent challenging features was evident as early as 80 years ago. In the 1930s, neurosurgeon Walter Dandy reported recurrence of contralateral GBM even after a hemispherectomy, 
emphasizing thus how infiltrative these tumors are. Patients with GBM exhibit a deficient anti-tumor immune response. Early reports of GBM patients with a postoperative surgical site infection who exhibit longer survival served as the first hint, sparking an interest in the topic. This phenomena was initially attributed to the non-specific immune recruitment invoked by the local bacterial lipopolysaccharide (LPS), targeting the tumor as well to some extent. Since these pivotal observations, a plethora of studies characterizing the immunosuppressive mechanisms in GBM have been conducted [3-19]. As discussed later, both arms of the immune system were shown to be hampered in GBM, namely the local cellular immunity mediated by the $\mathrm{Th}_{1}$ subset of helper $\mathrm{T}$ cells, and the systemic humoral immunity mediated by the $\mathrm{Th}_{2}$ subset of helper $\mathrm{T}$ cells $[7,8]$.

Immunotherapy is rapidly becoming one of the pillars of anti-cancer therapy. Its targeted nature and reduced treatment related toxicity, stemming from recruiting and activating own selective cytotoxic mechanisms, makes it intuitively an attractive option. GBM has not received similar clinical successes as of yet. This may be attributed to its relative inaccessibility (protected by the BBB), its poor immunogenicity, few characterized cancer antigens, or any of the many other immune mechanisms known to be hampered, briefly discussed next [3-19]. Preclinical studies suggest that immunotherapies can elicit significant anti-tumor responses in GBM, overcoming some of the barriers and tumor-related escape mechanisms, while others fail at specific points. Moreover, immunotherapies may have the potential to work synergistically with other treatment modalities such as high-intensity focused ultrasound (HIFU), surgery, radiotherapy, or alkylating agents.

Continuous-wave $(\mathrm{CW})$ high-intensity focused ultrasound (HIFU) is emerging as a promising treatment approach. CW-HIFU is the only noninvasive thermal technique that allows for real-time imaging of the treatment progress using MR thermometry [20]. High-energy ultrasound beams are applied to focus acoustic energy on a well-defined region. Although, single ultrasound beams penetrate a tissue without causing any significant heat, focusing beams from multiple directions into a selected region results in a temperature rise (to $>60{ }^{\circ} \mathrm{C}$ ), and subsequently inducing coagulative necrosis [20-23]. Ultrasonic energy emitted from concave piezoelectric ceramics can be tightly focused with radial and axial dimensions of only $1-2$ and $10-20 \mathrm{~mm}$, respectively, based on the range of frequencies and transducer geometry. Complete tumor ablation is then achieved by employing multiple stereotactic sonications [20, 23-25].

The effects of FUS on the tissue are not merely thermal. Mounting evidence suggests that in addition to the thermal ablation mechanism, HIFU induces mechanical acoustic cavitation which has both mechanical and molecular implications and also boosts the host anti-tumor immune responses (Table 1) [26-47]. We will briefly review separately the different pertinent immunosuppressive mechanisms harnessed by GBM and the immunomodulatory effects of HIFU. Of note, many such HIFU-related mechanisms were shown for non-GBM tumors. We will next attempt at offering a conceptual synopsis of the synergistic treatment of GBM utilizing HIFU and immunotherapy.

\section{Pertinent facts in immunology and brain-cancer immunotherapy}

The initial interaction of the $\mathrm{T}$ cell receptor (TCR) with the major histocompatibility complex (MHC)-peptide complex is stabilized by the binding of $\mathrm{CD}^{+}$or $\mathrm{CD}^{+}$ co-receptors molecules (termed as signal 1) [48]. This interactions are accompanied by the interaction of other receptors (termed as co-stimulatory or co-inhibitory signals), a process termed as signal 2 . Efficient $\mathrm{T}$ cell activation requires as little as a single MHC-peptide complex for $\mathrm{CD}^{+} \mathrm{T}$-helper cells and as little as 10 for cytotoxic $T$ cells (CTLs) [49], yet a signal 1 interaction that lacks a stimulatory signal 2 will fail to elicit $\mathrm{T}$ cell activation and differentiation. Different co-stimulatory (e.g., CD28) and co-inhibitory (e.g., CTLA-4) signal 2 molecules directs differentiation to different avenues, ranging from a potent anti-tumor response (in CD28 activation), to anergy and even apoptosis (upon CTLA-4 or Fas activation) [48]. A local cytokine based pro-inflammatory or antiinflammatory milieu plays a significant role in driving the immune response as well.

Mounting an effective brain anti-tumor immune response requires that certain requirements are met. As discussed later, the GBM cells developed mechanisms to evade or block every one of the following: first, the effector $\mathrm{T}$ cells, antibodies, or cytokines must penetrate the brain parenchyma before reaching the tumor bed. This entails crossing in active state through the $\mathrm{BBB}$ [50]. Some authors claim that the BBB is compromised/ disrupted in the vicinity of the GBM [9] while others consider it a potent obstacle. Next, tumor-associated target antigens must be sufficiently different from selfantigens. Third, tumor cells must express sufficient MHC molecule to mount a specific CTLs effectormediated response. Fourth, a local inflammatory response should then be instigated and properly regulated. Fifth, CTLs and $\mathrm{T}_{\mathrm{H}} 1$ cells must retain their anti-tumor effector function during migration through the brain parenchyma and its resident cells. The local cytokine microenvironment should support the $\mathrm{T}$ cell function. Finally, effector cell functionality must be retained during the encounter with the tumor cells, not suppressed actively or passively by the tumor cells. 
Table 1 Focused ultrasound immunomodulatory effect-literature review

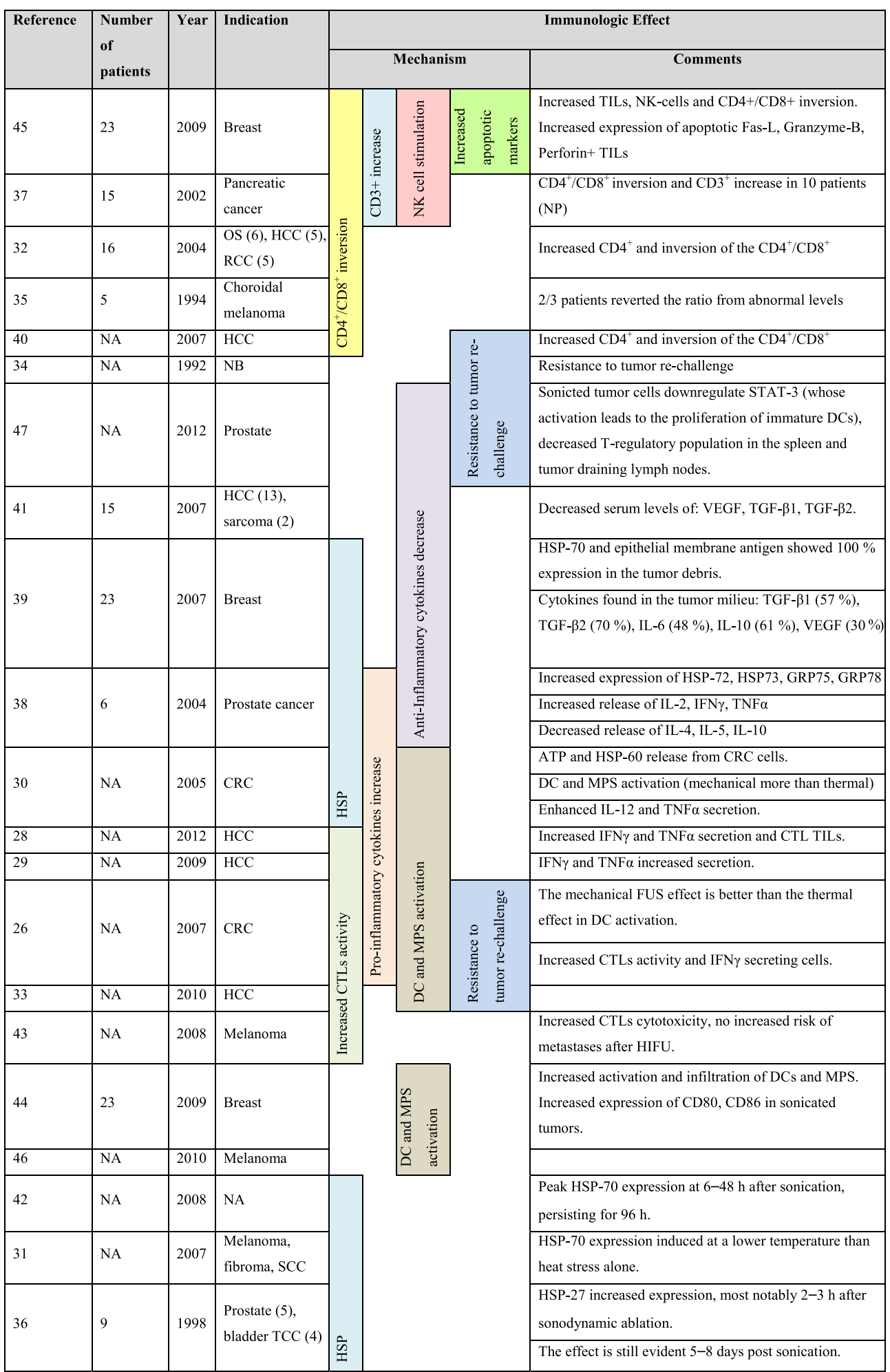

$N A$ not applicable, usually refers to pre-clinical studies, $N B$ neuroblastoma, $C D$ cluster of differentiation, $N P$ not statistically significant, $O S$ osteosarcoma,

$H S P$ heat shock proteins, $T C C$ transitional cell carcinoma, $H C C$ hepatocellular carcinoma, $R C C$ renal cell carcinoma, $C R C$ colorectal carcinoma, $D C$ dendritic cells, MPS mononuclear phagocyte system (i.e.,macrophages), CTLs cytotoxic (CD8 ${ }^{+}$) T lymphocytes, SCC squamous cell carcinoma, TILs tumor infiltrating lymphocytes. 
Immune mechanisms hampered in GBM

A multitude of immunosuppressive mechanisms (both active and passive) as well as immune-evasion techniques are attributed to GBM cells (refer to Fig. 1) [3-19]. GBM patients were shown to exhibit overall low number of circulating $\mathrm{T}$ cells, rendering them vulnerable to any systemic challenge (infection, allergic reaction, or response to tumors) [10]. Fecci et al. [11] noted an abnormally high proportion ( $>2.5$-fold increase) of circulating inhibitory $\mathrm{T}_{\text {reg }}$ cells $\left(\mathrm{CD}^{+}{ }^{+}\right.$Fox $\left.\mathrm{P}^{+}\right)$populations in GBM patients, unrelated to the co-administration of glucocorticoids [11]. The relative proportion of circulating $\mathrm{T}_{\text {reg }}$ cells is known to linearly correlate with in vitro suppression of effector T cell activation [12]. The most common genetic alteration in GBM (occurring in 80 95 \% of tumors) is the loss of heterozygosity in chromosome \#10 [7, 8, 13, 14]. Loss of the PTEN gene (phosphatase and tensin homologue) in this affected locus (10q23.3), serving as an inhibitor of the Phospho-inositol-3 kinase-signalling pathway may mediate a decrease in tumor cell immunogenicity by increasing the expression of B7-H1 (a coinhibitory signal 2 molecule discussed previously), as well as increases anti-inflammatory $\left(\mathrm{T}_{\mathrm{H}} 2\right.$ type) cytokines release $[13,14]$. These mechanisms independently support the evolution of anergy and tolerance to the tumor.
Multiple genetic pathways, involved in cell-cycle regulation, growth-factor receptors presentation, and cytokine formation and regulation are known to be affected in GBM. Such pathways include the p16/pRb/CDK4 pathway, the p53/MDM2/p14ARF pathway, the epidermal growth factor receptor (EGF-R) gene [with unique variants like the EGFRvIII, responsive to Erlotinib], platelet-derived growth factor receptor (PDGF-R), and PI3-kinase/PTEN cascades [13, 14]. An immune-evasion mechanism suggested is the tumor's ability to downregulate or express low levels of class-I MHC [15], hiding its existence from the cellular arm of the immune system, thus not detected by $\mathrm{CD} 4^{+} \mathrm{T}$ helper cells or $\mathrm{CD} 8^{+} \mathrm{CTLs}$. This feature of immune evasion is compounded by the GBM ability to express aberrant non-classical MHC class I molecules (class Ib) termed as HLA-G. These decoy MHC molecules are structurally related to the classical MHC molecules (the class-Ia composed of HLA-A, HLA-B, HLA-C). HLA-G expression, renders tumor cells highly resistant to direct CTL-mediated alloreactive lysis, inhibits the alloproliferative response, and prevents efficient priming of cytotoxic T cells [16].

Additional GBM-related mechanisms involve the upregulation of anti-apoptotic proteins by the GBM tumor cells [17], rending the tumor cells immortal (e.g.,

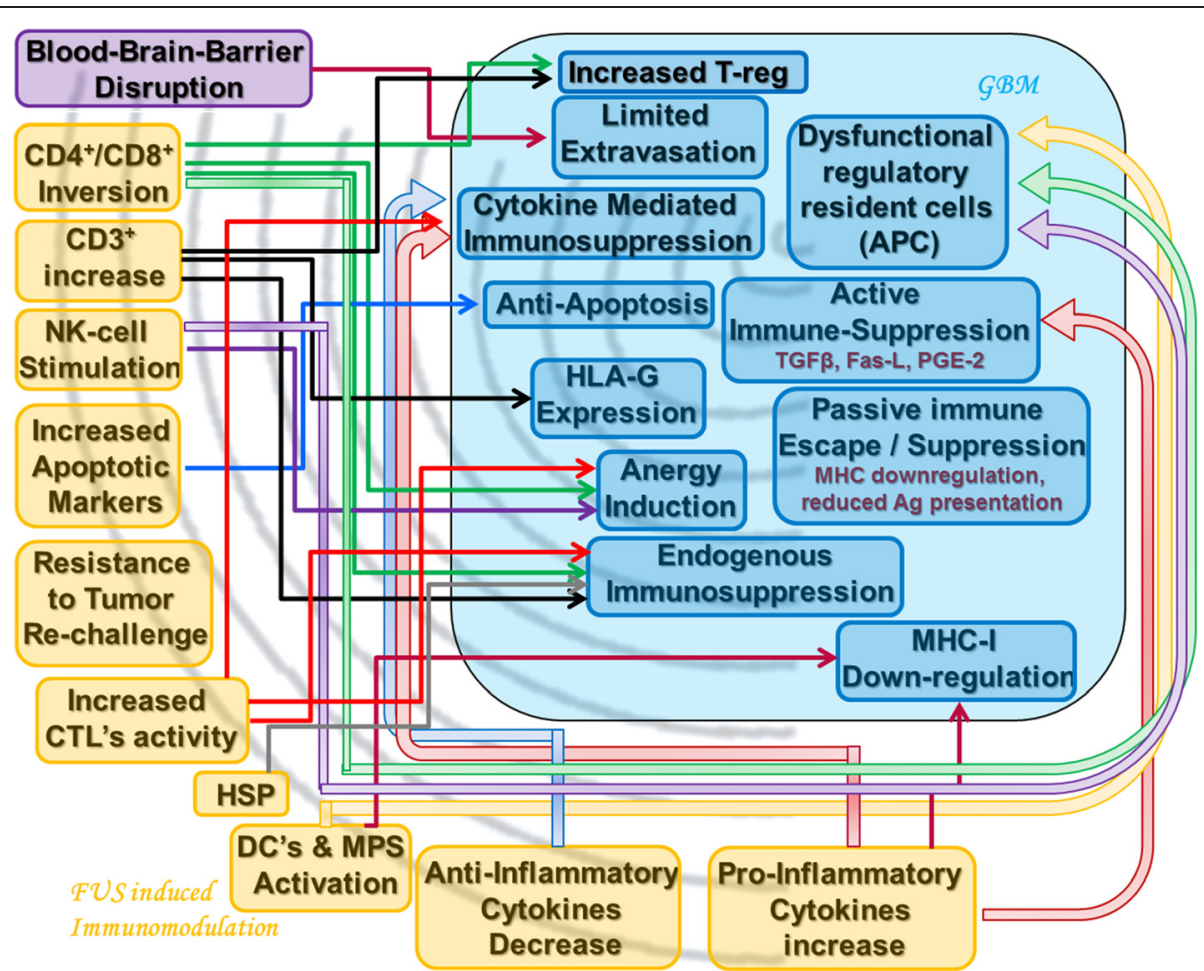

Fig. 1 Synopsis. The key established mechanisms hampered in GBM and the key immune-modulating effects of HIFU are presented. A theoretical action-reaction scheme is presented, connecting certain known GBM-evasion mechanisms with the FUS-induced counter response. Note that a single FUS-induced effect may influence a multitude of immunosuppressive mechanisms and vice versa. Refer to text 
unresponsive to death signals). Synergistically with all mentioned is the tumor-induced, cytokine driven, local immunosuppressive microenvironment. Reduced levels of pro-inflammatory cytokines (those driving to the $\mathrm{T}_{\mathrm{H}} 1$ differentiation) and increased local concentrations of anti-inflammatory cytokines and mediators (those driving to the $\mathrm{T}_{\mathrm{H}} 2$ differentiation) drive both the tissue and immune-elements that manage to reach the tumor site into an inactive or dysfunctional state [18]. Cytokines secreted, be it directly or indirectly by the GBM tumor cells, mediate immune-anergy and tumor proliferation $[10,19]$. Of note, the complex interplay between the different mechanisms stated is complex and largely unknown.

\section{FUS-induced anti-tumor effects}

Focused ultrasound exerts an anti-tumor effect utilizing three distinct complementary "modes" of action: thermal ablation, acoustic cavitation, and immunomodulation. Thermal ablation, the most obvious and characterized mode, is similar to many other lesional technologies in that the tissue at the focal point is targeted with the goal of causing coagulative necrosis. The second mechanism, acoustic cavitation, is in essence mechanical lysis of the tissue caused by harnessing acoustic cavitation, namely the rapid expansion and contraction of microbubbles/ gaseous nuclei in cells through acoustic pressure within the targeted tissue [51]. This process enhances the local heating [52] as well as leads to collapse of the intracellular machinery (i.e., mitochondria, endoplasmic reticulum, as well as nuclear and cell membranes) [20]. Harnessing the advantages of mechanical cavitation, the FUS aided disruption of the BBB (at lower exposure) serves as another synergistic mechanism to prime an anti-GBM response (discussed next) [53, 54]. In addition to disrupting the $\mathrm{BBB}$, these micro-bubbles can potentially be loaded with tumor antigens, allowing for immune-homing, mounting a more focused, effective immune response [53-55]. The third mechanism employs sub-lethal mild hyperthermia, i.e., a uniform low-level heating of a region of interest [39, 56, 57]. Pulsed-mode HIFU with negative pressures equal or higher than that used for thermal ablation (7-12 MPa) was shown to boost the systemic anti-tumor immune response through multiple mechanisms (Table 1) [26-47]. Immunologically speaking, although considered different, each of these modes shares a final common pathway in terms of initiating an immune response. This response is instigated through the release of tumor debris rich in unique antigens and danger signals into the inter-cellular matrix [51].

\section{FUS-induced immuno-modulating effects}

Many preclinical and clinical studies have demonstrated that FUS can facilitate and amplify an immune anti- tumor response, prolonged overall survival and protection from growth of new tumors when re-challenged (Table 1) [26-47]. One should note that all immunemodulating effects discussed hereafter were described on other tumor types. The assumption that these effects are FUS/HIFU based and are tumor type independent, was not validated objectively. All three HIFU-related modes of action can be assigned an immune-modulating mechanism. The HIFU-induced thermal ablation causes surviving tumor cells to upregulate danger signals such as heat shock proteins (HSP) and adenosine triphosphate (ATP). HSPs have long been viewed as potent innate immunity tools, serving to increase the tumor immunogenicity $[30,31,58]$. Second, mechanical cavitation allows for better $\mathrm{BBB}$ penetration for antigens and immune cells as well as results in lysis-related tumor debris. These debris serve then as potent antigens for the immune system, activating dendritic cells (DCs) [29]. Third, FUS mitigates tumor-induced immunosuppression. We will now briefly discuss each of the last two proposed mechanisms. A thorough discussion of the direct ablative thermal effect is not in the scope of this review. Table 1 presents a brief overview of key preclinical and clinical studies, per different tumor type, segregated based on the proposed FUS-induced immunomodulatory effect. Of note, this table is not restricted to studies conducted in GBM or in the CNS, rather serves as a proof of concept for FUS-based immunetriggered events.

\section{Microbubbles and BBB disruption}

The addition of microbubbles (MB) to FUS can help generate more local heating in the area of focus [52], but at lower exposures, FUS with MB can be used solely for BBB disruption. These microbubbles, delivered intravenously, are composed of lipid-encased perfluorocarbon gas, and measure approximately 1-5 $\mu \mathrm{m}$ in diameter [59]. Recent reports suggest that the permeability of the blood vessels can be substantially increased by FUS in the presence of MB. Even at greatly reduced acoustic pressures, barely above the pressure thresholds employed in diagnostic ultrasound, these $\mathrm{MBs}$ can be used to enhance targeted delivery of chemotherapeutic agents [60-63]. FUS exposure bursts of $10 \mathrm{~ms}$, repeated at a frequency of $1 \mathrm{~Hz}$ and used for 20-30 s durations are the typical settings used. FUS with MB impose mechanical forces on the vascular endothelium which then results in a transient opening of the inter-endothelial tight junctions [64]. This translates to a local and reversible BBB disruption. The size and resonance frequency of the microbubbles can be altered, allowing for agents up to $2000 \mathrm{kDa}$ to enter, in order to facilitate different goals [65]. Larger microbubbles require less acoustic pressure to achieve BBB opening. The FUS and MB BBB-disruption effects 
last for several hours and can be localized to the tumor region, prior to returning to the pre-FUS state [66]. Dynamic contrast-enhanced MRI has been shown to be able to monitor the kinetics of BBB disruption [67].

Several preclinical studies have demonstrated the feasibility of BBB disruption by FUS and MB for the administration of chemotherapeutics in the treatment of glioma. Aryal et al. [68] demonstrated increased survival in a rat glioma model, upon treatment with a combination of FUS and liposomal doxorubicin. Such liposomal doxorubicin would not cross the BBB without FUS-mediated disruption. Liu et al. [69] reported an increased concentration of temozolomide in a glioma model after FUS, which correlated with a diminished tumor progression and increased animal survival [69]. Yang et al. [70] reported the successful use of HIFU in delivering interleukin-4 (IL-4) receptor-targeted liposomal doxorubicin for enhanced targeted drug delivery and antitumor effect in GBM mouse model [70]. FUS with MB method of BBB disruption was shown to be applicable in delivering nanoparticles, DNA, plasmid vectors, and antibodies [71, 72].

Chen et al. [73] reported their successful preclinical feasibility study of FUS-induced BBB disruption in order to enhance IL-12 delivery in a C-6 glioma rat model. FUS-induced BBB opening had no obvious effect on the $\mathrm{T}$ lymphocytes population in normal animals, either in the brain or systemically. Yet, it triggered mild changes in the tumor-infiltrating lymphocyte (TIL) population, particularly in numbers of $\mathrm{CD}^{+} \mathrm{CD}^{+}$CTLs in the tumor region. IL-12 administration triggered a profound increase in all TIL populations, including $\mathrm{CD}^{+} \mathrm{CD} 4^{+} \mathrm{T}$ helper cells, CTLs, and $\mathrm{CD} 4^{+} \mathrm{CD} 25^{+} \mathrm{T}_{\text {reg. }}$. The combined FUS-BBB opening with IL-12 administration produced the most significant IL-12 increase, CTL increase and $\mathrm{CTL} / \mathrm{T}_{\text {reg }}$ ratio increase, thus contributing to the most significant suppression of tumor progression and increased animal survival. These reports provided evidence that FUS-mediated BBB opening can enhance immunemodulating agent delivery to the brain.

\section{Cytokines and stress response}

HSPs are intracellular molecular chaperones, able to bind tumor peptide antigens and enhance tumor cell immunogenicity [74-79]. Antigen-presenting cells (APCs; namely DCs, macrophages and $\mathrm{CD} 4^{+} \mathrm{T}$ helper cells) endocytose the HSP-tumor peptide complex and present the chaperoned peptides directly to tumor-specific CTLs. This evokes potent cellular immune responses against tumor cells [74-79]. Autologous HSP-peptide complexes generated from a single individual tumor were shown to generate a therapeutic immune response in animal models [39]. Because random mutations in GBM cells usually produce patient-unique tumor associated antigens, HSP vaccination may be a rationally personalized approach that may obviate the requirement to identify the unique antigens [74-79].

HIFU was shown to upregulate the expression of HSP70 both in vitro and ex vitro [30, 31, 42]. An increased HSP-70 expression was detected on the surviving cell membrane of 23 patients with breast cancer treated with HIFU ablation. HSP expression was mainly found in the central necrosis zone, with only sparse positively stained cells observed in the periphery [39]. The most striking change noted was the positive expression of EMA and HSP-70 on the treated cancer cells in all 23 patients after HIFU ablation [39]. EMA is a known mucinous glycoprotein considered as a differentiation tumor marker and a histological prognostic agent.

\section{Peripheral and intra-tumoral immune cell populations}

FUS was shown to enrich the TILs population in immune-potent pro-inflammatory potent anti-tumor effector cells. In human breast cancer specimens collected 1-2 weeks after FUS treatment [45], a significant increase in TILs of both T and B subsets at the margin of the ablated region was shown, as compared to FUSuntreated tumor samples. Immunohistochemistry analysis showed that a subset of these cells was activated CTLs $\left(\mathrm{CD} 57^{+}\right)$, expressing perforin and granzyme B molecules, indicative of a cytotoxic effector function [45]. A randomized study of 48 patients comparing those who underwent FUS treatment prior to radical mastectomy to patients who underwent surgery alone showed that those who had received FUS treatment prior to surgery had a significantly higher level of TILs of T, B, and NK cells subsets [80].

This TILs enrichment phenomenon is not limited to breast cancer. It was shown in patients treated with FUS suffering posterior uveal melanoma [35], pancreatic carcinoma [37], osteosarcoma [32], hepatocellular carcinomas (HCC) [32], and RCC [32]. Some combination of increased percentages of $\mathrm{CD}^{+} \mathrm{T}$ cells, $\mathrm{CD}^{+} \mathrm{T}$ cells, a higher $\mathrm{CD}^{+} / \mathrm{CD}^{+}$ratio, or NK cell stimulation [37] were observed [32, 35]. Refer to Table 1 for review of landmark studies and related mechanisms.

\section{Augmentation of dendritic cell activity}

FUS was shown to enhance the infiltration capabilities of dendritic cells (DCs) $[15,18]$ as well as other antigen presenting cells [44] in the treated tumor. Enhanced infiltration was followed subsequently with DCs migration to the draining lymph nodes, presenting tumorassociated antigens to a wide variety of circulating $\mathrm{T}$ cells [26, 43]. Zhang et al. [33] demonstrated that tumor debris induced by HIFU could serve as an effective immunogenic vaccine. This vaccine was shown to elicit tumor-specific immune responses, including induction of CTL cytotoxic activity, DCs-enhanced activation, and 
to protect naïve mice against a lethal tumor challenge. When tumor debris were loaded with immature DCs, this vaccine could induce maturation of DCs to a significant extent as well as increased CTLs cytotoxicity (manifested by elevated TNF- $\alpha$ and IFN- $\gamma$ secretion) [29]. The authors noted that while this vaccine was able to initiate a host specific immune response after $\mathrm{H} 22$ challenge in the vaccinated mice which resulted in tumor growth rate reduced, no survival advantage could be observed [29]. Thus, HIFU induces activation and stimulation of various APCs, leading to an increased expression of costimulatory molecules and enhanced secretion of IL-12 (via DCs) and TNF- $\alpha$ (macrophages) [30]. The potency of dendritic cell infiltration and activation was shown to be further improved when sparse-scan mode FUS was used compared to dense-scan mode [46, 53], yet a detailed technical and physical discussion of FUS modes is beyond the scope of this review.

\section{Resistances to tumor re-challenge and increased CTLS potency}

A portion of the immunomodulatory effects of FUS noted in different reports are lacking exact molecular mechanisms as of yet, collectively termed as resistance to tumor re-challenge. Some overlap with CTLs potentiation is suspected and hence these mechanisms are discussed together. Zhang et al. [33] reported their results with mice implanted with an HIFU-treated H22 hepatocellular carcinoma (HCC) tumor cells. The animals received a subcutaneous tumor challenge 10 days after vaccination. Tumor growth was significantly delayed in mice vaccinated with previously HIFU-treated tumor cells. Mice that were treated with HIFU showed an $88 \%$ survival rate at 60 days as compared to 36 and $16 \%$ for the sham-HIFU and control groups, respectively [33, 46]. Survival, however, was not different between the groups [33]. Other mouse models concurred with this observation and have shown a significant decrease in the growth rate of tumors during a second challenge [34]. Yang et al. [34] reported the use HIFU to treat C1300 neuroblastoma cells implanted in mouse flanks, followed by the re-challenge of the same tumor cells. The authors noted a significantly slower growth of re-implanted HIFU-treated tumors compared with the controls.

Several reports of potentiated CTLs effector activity and potency after HIFU support this mechanism (Table 1). Increased effector function can be measured by increased IFN $\gamma$ and TNF $\alpha$ secretion $[26,28,29]$ or increased direct CTLs-mediated cytotoxicity [43]. Xia et al. [28] reported that the cytotoxicity of CTLs and the number of activated tumor-specific CTLs was significantly increased in the H22 tumor bearing mice treated with HIFU. Adoptive transfer of the activated lymphocytes was shown to provide better long-term survival and lower metastatic rates in the mice re-challenged by the same tumor cells as compared with sham-HIFU and control groups, due to the induced anti-tumor cellular immunity in the mice [28]. Similar results were reported in mice implanted MC-38 colorectal adenocarcinoma (CRC) and melanoma after HIFU ablation. HIFU treatment was shown to induce an enhanced CTLs activity in vivo, providing protection against subsequent tumor re-challenge [26, 43].

\section{Synopsis and future directions}

In an attempt to direct hypothesis-based approach to FUSinduced immunomodulation in GBM, the key established mechanisms hampered in GBM and the key immunemodulating effects of FUS discussed in this review are presented in Fig. 1. A theoretical action-reaction scheme is presented; connecting certain known GBM-evasion mechanisms with the FUS-induced counter response. A single FUS-induced effect may influence a multitude of immunosuppressive mechanisms and vice versa. This scheme is given in an attempt to present a theoretical basis for the effectiveness of HIFU immunomodulation and synergistically supporting immunotherapies overcoming many of the GBM mediated immune-resistance mechanisms.

Future research still needs to be done to both dissect the different FUS-induced molecular and immunological mechanisms at play. Further research is needed to optimize the FUS treatment method, find other combinational therapies, defining how to most effectively use it in combination with immunotherapy [51].

\section{Conclusions}

No single treatment modality will cure GBM. It is increasingly apparent that surgery alone, HIFU ablation, chemotherapy, or immunotherapy will not solely triumph. We reviewed the FUS-induced immunomodulation effect, which can be harnessed to the current and developing immunotherapies approaches. Together, these modalities may overcome GBM-induced immune-evasion and generate a clinically relevant anti-tumor immune response. Further study to the synergistic collaboration of different therapeutic approaches and the elaborated molecular immune interplay will shed light on this formidable challenge.

\footnotetext{
Abbreviations

APCs: antigen-presenting cells; ATP: Adenosine triphosphate; BBB: bloodbrain barrier; CD: cluster of differentiation; CRC: colorectal adenocarcinoma; CTLA4: cytotoxic T-lymphocyte-associated protein 4; CTLs: cytotoxic T cells; CW: continuous-wave; DCs: dendritic cells; EGFR: epidermal growth factor receptor; FUS: focused ultrasound; GBM: glioblastoma multiforme; HCC: hepatocellular carcinomas; HIFU: high intensity focused ultrasound; HLA: human leukocyte antigen; HSP: heat shock proteins; IFN: interferon; IL: interleukin; LPS: lipopolysaccharide; MB: microbubbles; MGMT: $0^{6}$ methylguanine-DNA methyltransferase; MHC: major histocompatibility complex; PTEN: phosphatase tensin; RCC: renal cell carcinoma; TCR: T cell receptor; $T_{H}$ : T helper cell; TILs: tumor-infiltrating lymphocytes; TNF: tumor necrosis factor.
} 


\section{Competing interests}

Dr. Xu has received funds from the Focused Ultrasound Foundation for nonstudy related research efforts. All other authors have no personal or institutional financial interest in drugs or materials in relation to this paper.

\section{Authors' contributions}

$\mathrm{OCl}$ reviewed the literature, formatted the tables and figures, and drafted the manuscript. ZX provided critique and reviewed the manuscript. JPS provided critique and helped draft the manuscript. All authors read and approved the final manuscript

\section{Acknowledgement}

None

Received: 13 November 2015 Accepted: 11 January 2016

Published online: 22 January 2016

\section{References}

1. Stupp R, Mason WP, van den Bent MJ, Weller M, Fisher B, Taphoorn MJ, et al. Radiotherapy plus concomitant and adjuvant temozolomide for glioblastoma. N Engl J Med. 2005;352:987-96.

2. Weller M, van den Bent M, Hopkins K, Tonn JC, Stupp R, Falini A, et al. EANO guideline for the diagnosis and treatment of anaplastic gliomas and glioblastoma. Lancet Oncol. 2014;15(9):e395-403.

3. Or cohen-inbar. Focused Neurosurgery book. Jaypee Brothers Publishers, 2015.

4. Krauze AV, Myrehaug SD, Chang MG, Holdford DJ, Smith S, Shih J, et al. A Phase 2 Study of Concurrent Radiation Therapy, Temozolomide, and the Histone Deacetylase Inhibitor Valproic Acid for Patients With Glioblastoma. Int J Radiat Oncol Biol Phys. 2015;92(5):986-92.

5. Wainwright DA, Chang AL, Dey M, Balyasnikova IV, Kim CK, Tobias A, et al. Durable therapeutic efficacy utilizing combinatorial blockade against IDO, CTLA-4, and PD-L1 in mice with brain tumors. Clin Cancer Res. 2014;20(20): 5290-301.

6. Castro MG, Baker GJ, Lowenstein PR. Blocking immunosuppressive checkpoints for glioma therapy: the more the Merrier! Clin Cancer Res. 2014; 20(20):5147-9.

7. Aghi MK, Batchelor TT, Louis DN, Barker FG, Curry Jr WT. Decreased rate of infection in glioblastoma patients with allelic loss of chromosome 10q J Neurooncol. 2009;93:115-20.

8. Parsa AT, Waldron JS, Panner A, Crane CA, Parney IF, Barry JJ, et al. Loss of tumor suppressor PTEN function increases B7-H1 expression and immunoresistance in glioma. Nat Med. 2007:13:84-8.

9. Walker PR, Calzascia T, Dietrich PY. All in the head: obstacles for immune rejection of brain tumours. Immunology. 2002;107(1):28-38.

10. Hao C, Parney IF, Roa WH, Turner J, Petruk KC, Ramsay DA. Cytokine and cytokine receptor mRNA expression in human glioblastomas: evidence of Th1, Th2 and Th3 cytokine dysregulation. Acta Neuropathol. 2002;103(2): $171-8$.

11. Fecci PE, Mitchell DA, Whitesides JF, Xie W, Friedman AH, Archer GE, et al. Increased regulatory T-cell fraction amidst a diminished CD4 compartment explains cellular immune defects in patients with malignant glioma. Cancer Res. 2006:66(6):3294-302

12. See AP, Parker JJ, Waziri A. The role of regulatory T cells and microglia in Glioblastoma associated immunosuppression. J Neurooncol. 2015;123(3): 405-12.

13. Choe G, Horvath S, Cloughesy TF, Crosby K, Seligson D, Palotie A, et al. Analysis of the phosphatidylinositol 3'-kinase signaling pathway in glioblastoma patients in vivo. Cancer Res. 2003;63(11):2742-6.

14. Ermoian RP, Furniss CS, Lamborn KR, Basila D, Berger MS, Gottschalk AR, et al. Dysregulation of PTEN and protein kinase B is associated with glioma histology and patient survival. Clin Cancer Res. 2002;8(5):1100-6.

15. Yang L, Ng KY, Lillehei KO. Cell-mediated immunotherapy: a new approach to the treatment of malignant glioma. Cancer Control. 2003;10:138-47.

16. Pistoia V, Morandi F, Wang X, Ferrone S. Soluble HLA-G: Are they clinically relevant? Semin Cancer Biol. 2007;17:469-79.

17. Das A, Tan WL, Teo J, Smith DR. Expression of survivin in primary glioblastomas. J Cancer Res Clin Oncol. 2002;128(6):302-6.

18. Gomez GG, Kruse CA. Mechanisms of malignant glioma immune resistance and sources of immunosuppression. Gene Ther Mol Biol. 2006;10(A):133-46.
19. Zisakis A, Piperi C, Themistocleous MS, Korkolopoulou P, Boviatsis El, Sakas DE, et al. Comparative analysis of peripheral and localised cytokine secretion in glioblastoma patients. Cytokine. 2007;39(2):99-105.

20. Kennedy JE. High-intensity focused ultrasound in the treatment of solid tumours. Nat Rev Cancer. 2005;5:321-7

21. Haen SP, Pereira PL, Salih HR, Rammensee HG, Gouttefangeas C. More Than Just Tumor Destruction: Immunomodulation by Thermal Ablation of Cancer. Clin Dev Immunol. 2011;1-19.

22. Fischer K, Gedroyc W, Jolesz FA. Focused ultrasound as a local therapy for liver cancer. Cancer J. 2010;16(2):118-24.

23. Tempany CM, McDannold NJ, Hynynen K, et al. Focused ultrasound surgery in oncology: overview and principles. Radiology. 2011;259(1):39-56.

24. Wu F, Wang ZB, Chen WZ, Zou JZ, Bai J, Zhu H, et al. Advanced hepatocellular carcinoma: treatment with high-intensity focused ultrasound ablation combined with transcatheter arterial embolization. Radiology. 2005; 235(2):659-67

25. Wu F, Wang ZB, Zhu H, Chen WZ, Zou JZ, Bai J, et al. Feasibility of US-guided high-intensity focused ultrasound treatment in patients with advanced pancreatic cancer: initial experience. Radiology. 2005;236(3):1034-40.

26. Hu Z, Yang XY, Liu Y, Sankin GN, Pua EC, Morse MA, et al. Investigation of HIFU-induced anti-tumor immunity in a murine tumor model. J Transl Med. 2007;5(1):34

27. Liu F, Hu Z, Qiu L, Hui C, Li C, Zhong P, et al. Boosting high-intensity focused ultrasound-induced anti-tumor immunity using a sparse-scan strategy that can more effectively promote dendritic cell maturation. J Transl Med. 2010;8:7

28. Xia JZ, Xie FL, Ran LF, Xie XP, Fan YM, Wu F. High-intensity focused ultrasound tumor ablation activates autologous tumor-specific cytotoxic T lymphocytes. Ultrasound Med Biol. 2012;38:1363-71.

29. Deng J, Zhang Y, Feng J, Wu F. Dendritic cells loaded with ultrasoundablated tumour induce in vivo specific antitumour immune responses. Ultrasound Med Biol. 2010:36(3):441-8.

30. Hu Z, Yang XY, Liu Y, Morse MA, Lyerly HK, Clay TM, et al. Release of endogenous danger signals from HIFU-treated tumor cells and their stimulatory effects on APCs. Biochem Biophys Res Commun. 2005;335(1):124-31.

31. Hundt W, O'Connell-Rodwell CE, Bednarski MD, Steinbach S, Guccione S. In Vitro Effect of Focused Ultrasound or Thermal Stress on HSP70 Expression and Cell Viability in Three Tumor Cell Lines. Acad Radiol. 2007:14(7):859-70.

32. Wu F, Wang ZB, Lu P, Xu ZL, Chen WZ, Zhu H, et al. Activated anti-tumor immunity in cancer patients after high intensity focused ultrasound ablation. Ultrasound Med Biol. 2004;30(9):1217-22.

33. Zhang Y, Deng J, Feng J, Wu F. Enhancement of antitumor vaccine in ablated hepatocellular carcinoma by high-intensity focused ultrasound. World J Gastroenterol. 2010;16(28):3584-91.

34. Yang R, Reilly CR, Rescorla FJ, Sanghvi NT, Fry FJ, Franklin Jr TD, et al. Effects of high-intensity focused ultrasound in the treatment of experimental neuroblastoma. J Pediatr Surg. 1992;27(2):246-50. discussion 250-1 (1992).

35. Rosberger DF, Coleman DJ, Silverman R, Woods S, Rondeau M, Cunningham-Rundles S. Immunomodulation in choroidal melanoma: reversal of inverted CD4/CD8 ratios following treatment with ultrasonic hyperthermia. Biotechnol Ther. 1994;5(1-2):59-68.

36. Madersbacher S, Gröbl M, Kramer G, Dirnhofer S, Steiner GE, Marberger M. Regulation of heat shock protein 27 expression of prostatic cells in response to heat treatment. Prostate. 1998;37(3):174-81.

37. Wang $X$, Sun J. High-intensity focused ultrasound in patients with late-stage pancreatic carcinoma. Chin Med J. 2002;115(9):1332-5.

38. Kramer G, Steiner GE, Gröbl M, Hrachowitz K, Reithmayr F, Paucz L, et al. Response to sublethal heat treatment of prostatic tumor cells and of prostatic tumor infiltrating T-cells. Prostate. 2004;58(2):109-20.

39. Wu F, Wang ZB, Cao YD, Zhou Q, Zhang Y, Xu ZL, et al. Expression of tumor antigens and heat-shock protein 70 in breast cancer cells after highintensity focused ultrasound ablation. Ann Surg Oncol. 2007;14(3):1237-42.

40. Zhou P, Fu M, Bai J, Wang Z, Wu F. Immune response after high-intensity focused ultrasound ablation for $\mathrm{H} 22$ tumor. Journal of Clinical Oncology, ASCO 2007 Annual Meeting Proceedings (Post-Meeting Edition). 2007;25(18S):21169.

41. Zhou Q, Zhu XQ, Zhang J, Xu ZL, Lu P, Wu F. Changes in circulating immunosuppressive cytokine levels of cancer patients after high intensity focused ultrasound treatment. Ultrasound Med Biol. 2008:34:81-7.

42. Kruse DE, Mackanos MA, O'Connell-Rodwell CE, Contag CH, Ferrara KW. Short-duration-focused ultrasound stimulation of Hsp70 expression in vivo. Phys Med Biol. 2008;53(13):3641-60. 
43. Xing $Y$, Lu X, Pua EC, Zhong P. The effect of high intensity focused ultrasound treatment on metastases in a murine melanoma model. Biochem Biophys Res Commun. 2008;375(4):645-50.

44. Xu ZL, Zhu XQ, Lu P, Zhou Q, Zhang J, Wu F. Activation of tumor-infiltrating antigen presenting cells by high intensity focused ultrasound ablation of human breast cancer. Ultrasound Med Biol. 2009:35(1):50-7.

45. Lu P, Zhu XQ, Xu ZL, Zhou Q, Zhang J, Wu F. Increased infiltration of activated tumor-infiltrating lymphocytes after high intensity focused ultrasound ablation of human breast cancer. Surgery. 2009;145(3):286-93.

46. Liu F, Hu Z, Qiu L, Hui C, Li C, Zhong P, et al. Boosting high-intensity focused ultrasound-induced anti-tumor immunity using a sparse-scan strategy that can more effectively promote dendritic cell maturation. J Transl Med. 2010;8(1):7.

47. Huang X, Yuan F, Liang M, Lo HW, Shinohara ML, Robertson C, et al. M-HIFU inhibits tumor growth, suppresses STAT3 activity and enhances tumor specific immunity in a transplant tumor model of prostate cancer. PLoS ONE. 2012;7(7):e41632

48. Schwartz RH. T cell anergy. Annu Rev Immunol. 2003;21:305-34.

49. Irvine DJ, Purbhoo MA, Krogsgaard M, Davis MM. Direct observation of ligand recognition by T cells. Nature. 2002;419:845-9.

50. Rubin LL, Staddon JM. The cell biology of the blood-brain barrier. Annu Rev Neurosci. 1999;22:11-28.

51. Wu F. Heat-Based Tumor Ablation: Role of the Immune Response. Adv Exp Med Biol. 2016;880:131-53.

52. Kim YS, Rhim H, Choi MJ, Lim HK, Choi D. High-intensity focused ultrasound therapy: An overview for radiologists. Korean J Radiol. 2008;9(4):291-302.

53. Liu HL, Hsieh HY, Lu LA, Kang CW, Wu MF, Lin CY. Low-pressure pulsed focused ultrasound with microbubbles promotes an anticancer immunological response. J Transl Med. 2012;10(1):221.

54. Alkins R, Burgess A, Ganguly M, Francia G, Kerbel R, Wels WS, et al. Focused ultrasound delivers targeted immune cells to metastatic brain tumors. Cancer Res. 2013;73(6):1892-9.

55. Unga J, Hashida M. Ultrasound induced cancer immunotherapy. Adv Drug Deliv Rev. 2014;72:144-53.

56. Skitzki JJ, Repasky EA, Evans SS. Hyperthermia as an immunotherapy strategy for cancer. Curr Opin Investig Drugs. 2009;10(6):550-8.

57. Partanen A, Yarmolenko PS, Viitala A, Appanaboyina S, Haemmerich D, Ranjan A, et al. Mild hyperthermia with magnetic resonance-guided highintensity focused ultrasound for applications in drug delivery. Int J Hyperthermia. 2012;28(4):320-36.

58. Hundt W, Steinbach S, Burbelko M, Kiessling A, Rominger M, O'ConnellRodwell CE, et al. Induction of luciferase activity under the control of an hsp70 promoter using high-intensity focused ultrasound: combination of bioluminescence and MRI imaging in three different tumour models. Technol Cancer Res Treat. 2011;10(2):197-210.

59. Rodriguez A, Tatter SB, Debinski W. Neurosurgical Techniques for Disruption of the Blood-Brain Barrier for Glioblastoma Treatment. Pharmaceutics. 2015; 7(3):175-87.

60. Frenkel V, Li KC. Potential role of pulsed-high intensity focused ultrasound in gene therapy. Future Oncol. 2006;2(1):111-9.

61. Hwang $J H$, Brayman AA, Reidy MA, Matula TJ, Kimmey MB, Crum LA Vascular effects induced by combined 1-MHz ultrasound and microbubble contrast agent treatments in vivo. Ultrasound Med Biol. 2005;31(4):553-64

62. Ferrara K, Pollard R, Borden M. Ultrasound microbubble contrast agents: fundamentals and application to gene and drug delivery. Annu Rev Biomed Eng. 2007;9:415-47.

63. Vestweber D. Relevance of endothelial junctions in leukocyte extravasation and vascular permeability. Ann N Y Acad Sci. 2012;1257:184-92.

64. McDannold NJ, Vykhodtseva NI, Hynynen K. Microbubble contrast agent with focused ultrasound to create brain lesions at low power levels: MR imaging and histologic study in rabbits. Radiology. 2006;241(1):95-106.

65. Chen H, Konofagou EE. The size of blood-brain barrier opening induced by focused ultrasound is dictated by the acoustic pressure. J Cereb Blood Flow Metab. 2014;34:1197-204.

66. Sheikov N, McDannold N, Vykhodtseva N, Jolesz F, Hynynen K. Cellular mechanisms of the blood-brain barrier opening induced by ultrasound in presence of microbubbles. Ultrasound Med Biol. 2004;30(7):979-89.

67. Yang FY, Ko CE, Huang SY, Chung IF, Chen GS. Pharmacokinetic changes induced by focused ultrasound in glioma-bearing rats as measured by dynamic contrast-enhanced MRI. PLoS ONE. 2014;9(3):e92910.
68. Aryal M, Vykhodtseva N, Zhang YZ, Park J, McDannold N. Multiple treatments with liposomal doxorubicin and ultrasound-induced disruption of blood-tumor and blood-brain barriers improve outcomes in a rat glioma model. J Control Release. 2013;169(1-2):103-11.

69. Liu HL, Huang CY, Chen JY, Wang HY, Chen PY, Wei KC. Pharmacodynamic and therapeutic investigation of focused ultrasound-induced blood-brain barrier opening for enhanced temozolomide delivery in glioma treatment. PLOS ONE. 2014;9(12):e114311.

70. Yang FY, Wong TT, Teng MC, Liu RS, Lu M, Liang HF, et al. Focused ultrasound and interleukin-4 receptor-targeted liposomal doxorubicin for enhanced targeted drug delivery and antitumor effect in glioblastoma multiforme. J Control Release. 2012;160(3):652-8.

71. Diaz RJ, McVeigh PZ, O'Reilly MA, Burrell K, Bebenek M, Smith C, et al. Focused ultrasound delivery of Raman nanoparticles across the blood-brain barrier: Potential for targeting experimental brain tumors. Nanomedicine. 2014;10(5):1075-87.

72. Alonso A. Ultrasound-induced blood-brain barrier opening for drug delivery. Front Neurol Neurosci. 2015;36:106-15.

73. Chen PY, Hsieh HY, Huang CY, Lin CY, Wei KC, Liu HL. Focused ultrasoundinduced blood-brain barrier opening to enhance interleukin-12 delivery for brain tumor immunotherapy: a preclinical feasibility study. J Transl Med. 2015;13:93.

74. Pockley AG. Heat shock proteins as regulators of the immune response. Lancet. 2003;362(9382):469-76.

75. Fučíková J, Bartůňková J, Špíšek R. The Concept of Immunogenic Cell Death in Antitumor Immunity. Klin Onkol. 2015;28(Supplementum 4):48-55.

76. Muth C, Rubner Y, Semrau S, Rühle PF, Frey B, Strnad A et al. Primary glioblastoma multiforme tumors and recurrence : Comparative analysis of the danger signals HMGB1, HSP70, and calreticulin. Strahlenther Onkol. 2015; [Epub ahead of print].

77. Wang X, Ji J, Zhang H, Fan Z, Zhang L, Shi L et al. Stimulation of dendritic cells by DAMPs in ALA-PDT treated SCC tumor cells. Oncotarget. 2015 [Epub ahead of print].

78. Todryk SM, Gough MJ, Pockley AG. Facets of heat shock protein 70 show immunotherapeutic potential. Immunology. 2003;110(1):1-9.

79. Bajzert J, Stefaniak T. Heat shock protein HSP60 and the perspective for future using as vaccine antigens. Postepy Hig Med Dosw (Online). 2015;69:1149-68.

80. Higgins JP, Bernstein MB, Hodge JW. Enhancing immune responses to tumor-associated antigens. Cancer Biol Ther. 2009;8(15):1440-9.

\section{Submit your next manuscript to BioMed Central and we will help you at every step:}

- We accept pre-submission inquiries

- Our selector tool helps you to find the most relevant journal

- We provide round the clock customer support

- Convenient online submission

- Thorough peer review

- Inclusion in PubMed and all major indexing services

- Maximum visibility for your research

Submit your manuscript at www.biomedcentral.com/submit 